\title{
Radiosensitivity of human ovarian cancer cells is enhanced by pseudolaric acid $B$ due to the inhibition of the Ras/Raf/ERK signaling pathway
}

\author{
QUQING SONG, SHENG JIANG, XINXIN ZHANG, CHUNXIA PAN, \\ CHUNHUA LU, JINGWEI PENG and QINGSHUI LI \\ Department of Gynecological Oncology, Shandong Cancer Hospital Affiliated to Shandong University, \\ Shandong Academy of Medical Sciences, Jinan, Shandong 250117, P.R. China
}

Received December 30, 2016; Accepted July 20, 2017

DOI: $10.3892 /$ etm.2017.5500

\begin{abstract}
Ovarian cancer has the highest mortality rate among gynecological cancers; the most effective therapy for this cancer is a combination of radiation treatment and chemotherapy. However, radiation resistance is the leading factor associated with treatment failure. The present study aimed to investigate pseudolaric acid $\mathrm{B}(\mathrm{PAB})$ as a potential radiosensitizer for the treatment of ovarian cancer. The present study performed MTT and clonogenic assays, and demonstrated that PAB could induce a radiosensitizing effect on SKOV-3 cells. An Annexin V/propidium iodide staining assay revealed that PAB exerted a radiosensitizing effect by inducing SKOV-3 cell apoptosis. In addition, western blot analysis demonstrated that the activity of the Ras/RAF proto-oncogene serine/threonine-protein kinase/extracellular signal-regulated kinase signaling pathway was reduced by combination therapy with $\mathrm{PAB}$ and irradiation. In conclusion, the present study establishes $\mathrm{PAB}$ as a radiosensitizer, and provides a rational basis for the use of $\mathrm{PAB}$ and irradiation as a combination therapy to treat ovarian cancer.
\end{abstract}

\section{Introduction}

Ovarian cancer presents as a complex cystic mass in the pelvis and is the leading cause of mortality amongst all types of gynecological cancer $(1,2)$. No anatomical barrier exists to prevent the widespread metastasis of ovarian cancer (3). Currently, the most effective therapy for this disease is cytoreductive surgery alongside chemotherapy regimens; these predominantly use

Correspondence to: Dr Qingshui Li, Department of Gynecological Oncology, Shandong Cancer Hospital Affiliated to Shandong University, Shandong Academy of Medical Sciences, 107 Jiyan Road, Jinan, Shandong 250117, P.R. China

E-mail: qingshui_li@126.com

Key words: pseudolaric acid $\mathrm{B}$, irradiation, apoptosis, ovarian cancer, Ras-Raf-ERK signaling pathway, radioresistance platinum analogues with the addition of a taxane and induce apoptosis to kill cancer cells $(3,4)$. However, the overall cure rate of patients with ovarian cancer remains low at $~ 30 \%$ and the survival rate at 5 years is $38 \%$, meaning a more effective therapy is urgently needed (3).

Ionizing radiation, including X-rays and $\alpha$-particles, is often used to treat a variety of types of cancer (5-7). The radiation can induce cell death through a variety of different mechanisms, including apoptosis, autophagy, necrosis and accelerated senescence (7). The type of radiation used, the dosage and the region of the body targeted all determine the type of cell death that occurs. When treating cancer with irradiation, the degree of carcinogenic risk and radioresistance must be taken into account. To help improve understanding of this, it has been reported that the combination of radiotherapy with a radiosensitizing agent was being investigated (8).

Pseudolaric acid B (PAB) is present in a traditional Chinese medicine named 'Tu-Jin-Pi', which is derived from Pseudolarix kaempferi Gordon (9). Previous studies have demonstrated that PAB can have a range of different pharmacological effects, including antifungal, antimicrobial, antifertility and cytotoxic effects (9-12). It has been reported that PAB possesses antitumor activity against an ovarian cancer cell line by inducing caspase-dependent apoptosis (13). However, few studies have focused on investigating the potential combination of radiotherapy and PAB.

The present study aimed to investigate the anticancer activity of $\mathrm{PAB}$, and the combination of $\mathrm{PAB}$ and irradiation as a therapy against human ovarian cancer cells. First, it was established that the combination of PAB with irradiation was a potential treatment against ovarian cancer cells. Secondly, the type of cell death taking place and the underlying mechanisms causing it were investigated using the ovarian cancer cell line SKOV-3.

\section{Materials and methods}

Reagents and chemicals. PAB was obtained from the National Institute for the Control of Pharmaceutical and Biological Products (Beijing, China) with a purity of $>98 \%$. PAB was dissolved in dimethyl sulfoxide (DMSO) to make a stock 
solution, which was then diluted using McCoy's 5A (modified) medium (Invitrogen, Thermo Fisher Scientific, Inc., Waltham, MA, USA) to a final concentration of DMSO of $<0.05 \%$, which was considered to have no detectable effects on cell growth and viability. Trypsin, EDTA, PBS, monodansylcadaverine (MDC), propidium iodide (PI) and Annexin V were all purchased from Sigma-Aldrich (Merck KGaA, Darmstadt, Germany). McCoy's 5A (modified) medium and fetal bovine serum (FBS) were purchased from Invitrogen (Thermo Fisher Scientific, Inc.). Primary antibodies directed against microtubule-associated proteins 1/2 light chain 3 (LC3-I/II), autophagy protein 5 (ATG5), $\beta$-actin, phosphorylated (p)-Ras, p-RAF proto-oncogene serine/threonine-protein kinase (Raf) and extracellular signal-regulated kinase (ERK) were obtained from Cell Signaling Technology, Inc. (Danvers, MA, USA). Secondary antibodies were also obtained from Cell Signaling Technology, Inc. All other chemical reagents used in the study were of analytical reagent grade.

Cells and culture. The human ovarian adenocarcinoma SKOV-3 and OVCAR-3 cell lines were obtained from the American Type Culture Collection (ATCC; Manassas, VA, USA). SKOV-3 cells are resistant to tumor necrosis factor-induced apoptosis, and to several cytotoxic drugs, including diphtheria toxin, cisplatin and Adriamycin (14). The cells were cultured in Invitrogen McCoy's 5A (modified) medium supplemented with $10 \% \mathrm{FBS}$ at a temperature of $37^{\circ} \mathrm{C}$ with $5 \% \mathrm{CO}_{2}$. The cells were passaged with $0.25 \%$ (w/v) trypsin- $0.53 \mathrm{mM}$ EDTA solution. The cells were assayed in the logarithmic growth phase.

Radiation. Cells were cultured at a density of $1.5 \times 10^{5}$ cells/well in a 6-well plate and pretreated with $\mathrm{PAB}$ at different concentrations $(2.5-10 \mu \mathrm{M})$ in a humidified atmosphere of $37^{\circ} \mathrm{C}$ with $5 \% \mathrm{CO}_{2}$ for $24 \mathrm{~h}$. Then, the cells were irradiated with an X-ray source using a MD2 Linear Accelerator (Primus; Siemens AG, Munich, Germany) at a dose of 0-10 Gy.

Cell viability assay. An MTT assay was performed to determine cell viability. The SKOV-3 or OVCAR-3 cells were seeded into $96-$ well plates at a density of $1 \times 10^{4}$ cells/well. The cells were treated with PAB $(2.5,5$ or $10 \mu \mathrm{M})$ for $24 \mathrm{~h}$, by which time they had attained $70-80 \%$ confluence. Then, the cells were irradiated at 4 Gy. Afterwards, $10 \mu$ l MTT solution (5 g/l) was added to each well and the plates were incubated at $37^{\circ} \mathrm{C}$ with $5 \% \mathrm{CO}_{2}$ for another $2 \mathrm{~h}$. The resulting crystals were dissolved in $150 \mu \mathrm{l}$ DMSO and the absorbance (A) of the wells was measured using a microplate reader. The cell growth inhibitory ratio (\%) was calculated as follows: $\left(\mathrm{A}_{490, \text { control }}-\mathrm{A}_{490, \text { sample }}\right) /\left(\mathrm{A}_{490, \text { control }}-\mathrm{A}_{490, \text { blank }}\right) \times 100$.

Clonogenic assay. Cells were seeded into 6-well plates at a density of $2 \times 10^{2}$ cells/well and cultured at $37^{\circ} \mathrm{C}$ in an atmosphere with $5 \% \mathrm{CO}_{2}$ for $12 \mathrm{~h}$. The cells were then treated with PAB (2.5-10 $\mu \mathrm{M})$ and/or irradiated (2-10 Gy). A total of 2 weeks later, the colonies were fixed with $4 \%$ paraformaldehyde for $20 \mathrm{~min}$ at room temperature, and stained with $0.2 \%$ crystal violet for $10 \mathrm{~min}$ at room temperature. The surviving fraction (\%) was calculated as follows: $\left(1-\mathrm{N}_{\text {irradiated colonies }} / \mathrm{N}_{\text {control colonies }}\right)$ x 100 . All data were fitted into the linear quadratic model using Graphpad
Prism software (version 5; GraphPad Software, Inc., La Jolla, CA, USA).

Observation of morphological changes. The cells were seeded in 6 -well plate at a density of $1.5 \times 10^{5}$ cells/well, cultured overnight, then irradiated at a dose of 4 Gy following PAB treatment (2.5-10 $\mu \mathrm{M})$. The cellular morphology was subsequently observed using a phase-contrast microscope (Olympus Corporation, Tokyo, Japan).

Cell autophagy assay. Cell autophagy was measured using Sigma-Aldrich MDC staining assay (cat. no. D4008; Sigma-Aldrich; Merck KGaA) according to the manufacturer's instructions. The cells were seeded at a density of $2 \times 10^{6}$ cells/well in a 6 -well plate. Once the cells reached $70-80 \%$ confluence they were treated with different doses of PAB $(2.5-10 \mu \mathrm{M})$ and irradiated at a dose of $4 \mathrm{~Gy}$. After $24 \mathrm{~h}$ the cells were washed, the staining buffer of $0.05 \mathrm{mM}$ MDC in PBS was added and the plates were incubated at $37^{\circ} \mathrm{C}$ for $30 \mathrm{~min}$. The samples were analyzed using a flow cytometer (BD Biosciences, Franklin Lakes, NJ, USA). Apoptosis ratios were analyzed by CellQuest software (version 3; BD Biosciences).

Cell apoptosis assay. Cell apoptosis was measured using an Annexin V/PI staining assay. The cells were seeded at a density of $2 \times 10^{6}$ cells in a 6 -well plate, and when the cells reached $80 \%$ confluence they were treated with different doses of PAB (2.5-10 $\mu \mathrm{M})$ and irradiated with $4 \mathrm{~Gy}$. After $24 \mathrm{~h}$ the cells were washed with PBS, collected and then suspended in a Sigma-Aldrich Annexin V binding buffer (Sigma-Aldrich; Merck KGaA) according to the manufacturer's instructions. Annexin V and PI (Sigma-Aldrich; Merck KGaA) were added to the sample and it was incubated for $30 \mathrm{~min}$ at $37^{\circ} \mathrm{C}$. The cell apoptosis rate was analyzed using a flow cytometer (BD Biosciences). Autophagy ratios were analyzed by CellQuest software (version 3; BD Biosciences).

Western blot analysis. The cells were seeded into 6-well plate at a density of $1.5 \times 10^{5}$ cell/well, cultured overnight and then treated with 2.5-10 $\mu \mathrm{M}$ PAB and 4 Gy radiation. After incubation for $24 \mathrm{~h}$, the cells were washed, collected and then lysed using radioimmunoprecipitation assay lysis buffer containing a protease inhibitor cocktail (cOmplete ${ }^{\mathrm{TM}}$, Mini, EDTA-free Protease Inhibitor Cocktail; Sigma-Aldrich; Merck KGaA). Protein concentration was determined using the bicinchoninic acid protein assay. Samples contain $30 \mu \mathrm{g}$ proteins were loaded into each lane of an $8-12 \%$ SDS-PAGE gel. Electrophoresis was performed at $120 \mathrm{~V}$ for $1 \mathrm{~h}$ to separate the proteins. The proteins were transferred onto Millipore Immobilon ${ }^{\circledR}$-P transfer membrane (EMD Millipore, Billerica, MA, USA) over $2 \mathrm{~h}$ at $100 \mathrm{~mA}$. The membrane was blocked using 5\% non-fat dry milk in PBS for $2 \mathrm{~h}$. The membranes were then probed with antibodies directed against LC3-I/II (cat. no. 3868, 1:1,000), ATG5 (cat. no. 12994, 1:1,000), p-Ras (cat. no. 3321, 1:1,000), p-Raf (cat. no. 9421, 1:1,000), p-ERK (cat. no. 4370, 1:1,000) and $\beta$-actin (cat. no. $4970,1: 1,000$ ) overnight at $4^{\circ} \mathrm{C}$. The membranes were incubated with horseradish peroxidase-conjugated anti-rabbit secondary antibodies (cat. no. 7074, 1:1,000) for 

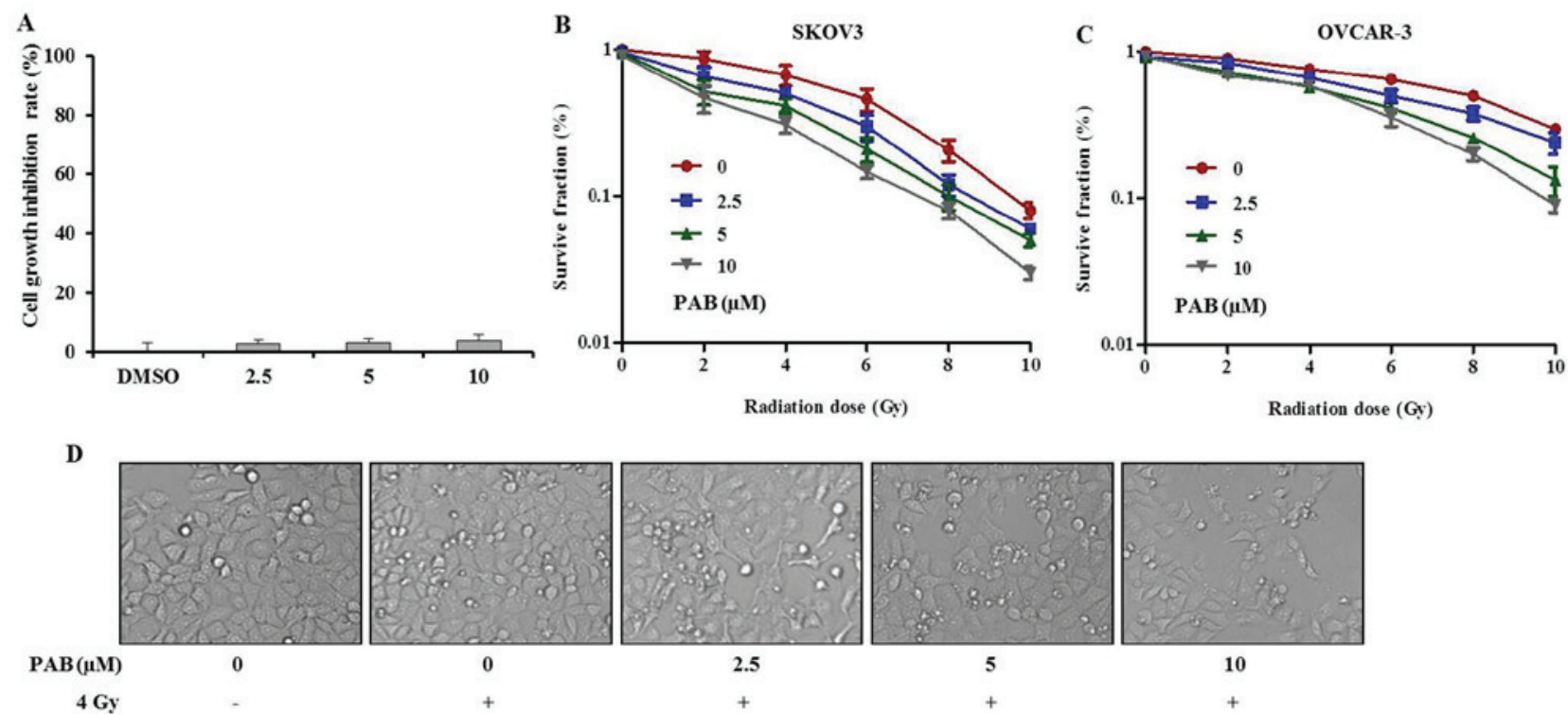

Figure 1. PAB treatment enhances the radiosensitivity of ovarian cancer cells. (A) Cells were treated with different doses of PAB for $24 \mathrm{~h}$ and then cell viability was measured by an MTT assay. (B) SKOV-3 or (C) OVCAR-3 cells were treated with different dose of PAB for $24 \mathrm{~h}$ and then treated with irradiation, the cell viability was subsequently measured using a clonogenic assay. The surviving fraction is expressed as a function of the irradiation dose. (D) Morphologic changes in SKOV-3 cells after PAB and irradiation treatment were observed. Magnification, x200. PAB, pseudolaric acid B.

$1 \mathrm{~h}$ at room temperature and the proteins were then detected using visualized by electrochemiluminescence $\left(\right.$ Pierce $^{\mathrm{TM}}$ ECL Plus Western Blotting Substate, cat. no. 32132; Thermo Fisher Scientific, Inc.). The films were scanned and quantified using ImageJ software (version 1.43; National Institutes of Health, Bethesda, MD, USA).

Statistical analysis. All results are expressed as the mean \pm standard deviation of at least three repeats. A one-way ANOVA followed by a Dunnett's post hoc test was performed to determine the statistical differences among groups using SPSS 17.0 software (SPSS, Inc., Chicago, IL, USA). P<0.05 was considered statistically significant. Four Gy radiation treatment group was considered as control group in all assays.

\section{Results}

$P A B$ enhances the radiosensitivity of ovarian cancer cells. To examine the effects of PAB on SKOV-3 cells, an MTT assay was performed (Fig. 1A). Increasing doses of PAB were administered for $24 \mathrm{~h}$ to detect the cytotoxicity of PAB. The results demonstrated that PAB had no significant inhibitory effect on the growth of ovarian cells.

Following this, a clonogenic assay was performed to investigate the effects of PAB on cell viability after irradiation. The surviving fractions of SKOV-3 and OVCAR-3 cells decreased as the doses of PAB and irradiation increased (Fig. 1B and C). Compared with OVCAR-3, SKOV-3 cells were more sensitive to radiation. Thus, SKOV-3 cells were used in subsequent assays. These results demonstrated that PAB acted in synergy with the irradiation, further reducing the surviving fraction of the cancer cells, when compared with treatment with irradiation alone. In addition, the morphological changes observed (Fig. 1D) were consistent with the results of the cell viability and clonogenic assays. These results indicate that
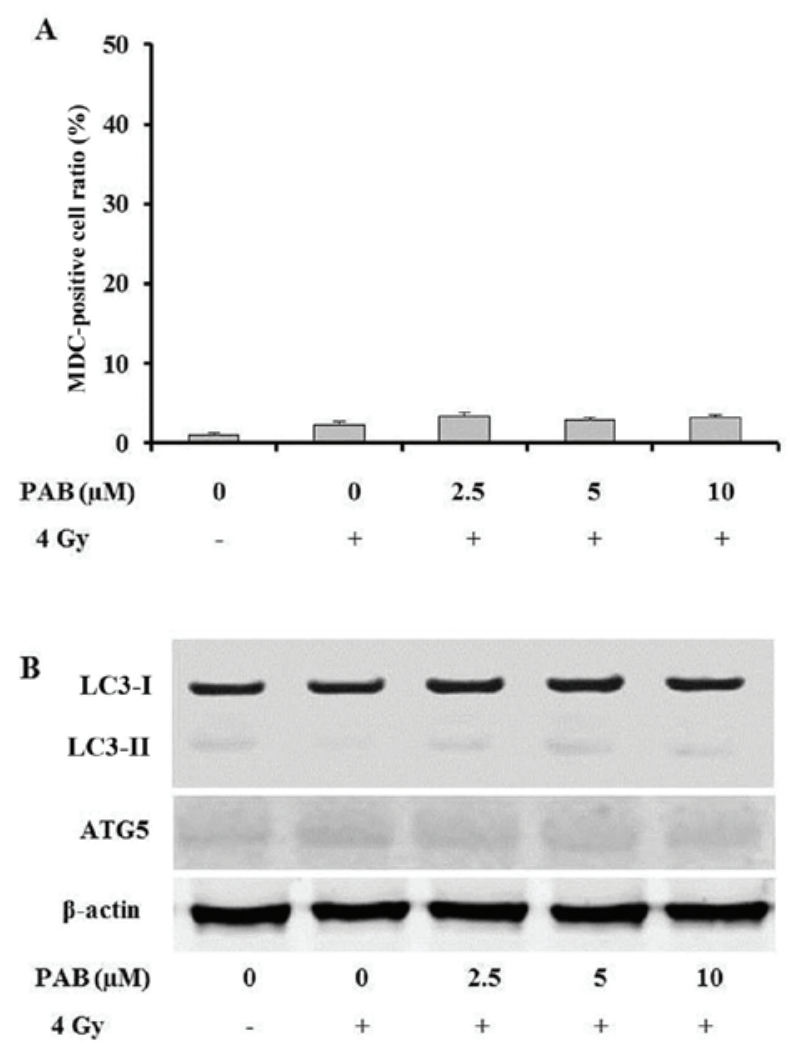

Figure 2. Autophagy is not affected by PAB in SKOV-3 cells. SKOV-3 cells were exposed to a range of $\mathrm{PAB}$ concentrations and $4 \mathrm{~Gy}$ radiation. After $24 \mathrm{~h},(\mathrm{~A})$ cells underwent MDC staining and the results were measured by flow cytometry or (B) the expression of LC3-I, LC3-II and ATG5 were analyzed by western blot analysis. PAB, pseudolaric acid B; MDC, monodansylcadaverine; LC3-I, microtubule-associated protein 1 light chain 3; LC3-II, microtubule-associated protein 2 light chain 3; ATG5, autophagy protein 5.

PAB may be a potent radiosensitizer and could have potential benefits in the treatment ovarian cancer. 
A
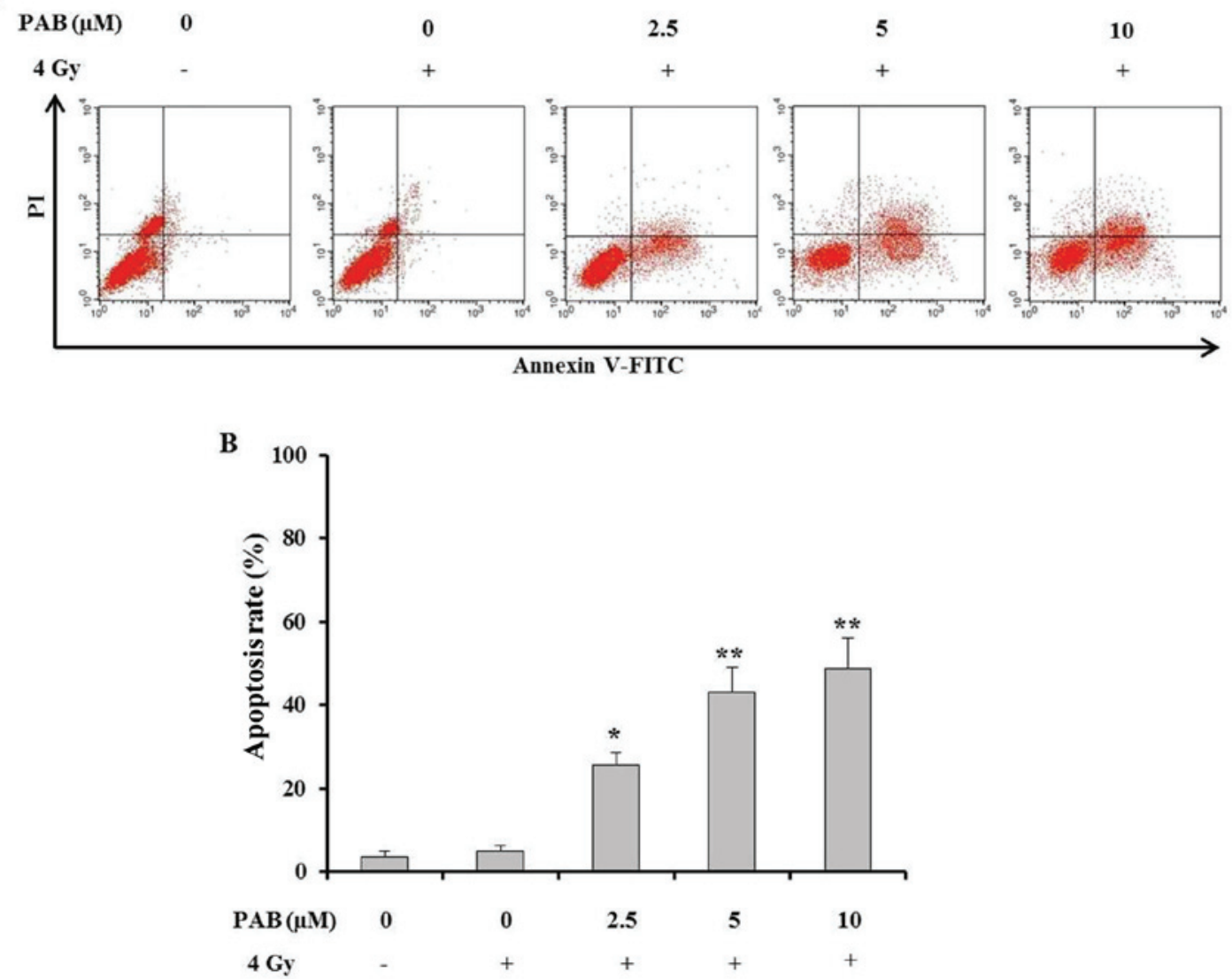

Figure 3. PAB dose-dependently increases the irradiation-induced apoptosis of SKOV-3 cells. SKOV-3 cells were exposed to a range of PAB concentrations and $4 \mathrm{~Gy}$ radiation. (A) After $24 \mathrm{~h}$, cells were subjected to Annexin V and PI staining and the results were measured by flow cytometry. (B) The percentage of apoptotic cells was quantified for each treatment. ${ }^{*} \mathrm{P}<0.05,{ }^{* * *} \mathrm{P}<0.01$ vs. 4 Gy radiation group. PAB, pseudolaric acid B; PI, propidium iodide; FITC, fluorescein isothiocyanate.

Autophagy is not involved in the radiosensitizing effects of PAB on SKOV-3 cells. To help determine the type of cell death undergone by SKOV-3 cells during combination treatment with PAB and irradiation, an MDC staining assay was performed to detect whether the cells underwent autophagy. The percentage ratio of MDC-positive cells was measured by flow cytometry. The results revealed that there were no notable changes in the level of autophagy following treatment with PAB (Fig. 2A). In order to confirm these findings, the expression of autophagy-associated proteins was also investigated. The expression of LC3-I, LC3-II and ATG5 exhibited no notable changes following PAB treatment (Fig. 2B). These results indicate that autophagy is not involved in the radiosensitizing effects of $\mathrm{PAB}$ in $\mathrm{SKOV}-3$ cells.

$P A B$ dose-dependently increases the irradiation-induced apoptosis of SKOV-3 cells. An Annexin V/PI staining assay was performed to determine the percentage of SKOV-3 cell death after treatment with a combination of PAB and irradiation (Fig. 3A). The results demonstrated that concurrent treatment with $\mathrm{PAB}$ and irradiation significantly increased the percentage of apoptotic SKOV-3 cells compared with the positive and negative controls (Fig. 3B). This effect increased with an increasing PAB dose. These results indicate that PAB dose-dependently enhances the irradiation-induced apoptosis of SKOV-3 cells and is an effective radiosensitizer.
$P A B$ and irradiation combination therapy inhibits the activation of the Ras-Raf-ERK signaling pathway. It has been reported that PAB could upregulate the expression of ERK in MCF-7 cells (9). To detect the underlying molecular mechanisms by which PAB radiosensitizes SKOV-3 cells, the effects of PAB and irradiation on the Ras-Raf-ERK signaling pathway were investigated. The expression of $\mathrm{p}$-Ras, $\mathrm{p}$-Raf and $\mathrm{p}$-ERK was dose-dependently decreased by treatment with PAB in combination with irradiation ( $\mathrm{P}<0.01$; Fig. $4 \mathrm{~A}$ and $\mathrm{B})$. In addition, the phosphorylation of these proteins was not inhibited by treatment with either irradiation (Fig. 4A and B) or PAB (Fig. 4C) alone, which was consistent with the apoptosis assay results. These results indicate that the Ras-Raf-ERK signaling pathway is inhibited by the combination treatment of PAB and irradiation, and that the disruption of this pathway can induce apoptosis.

\section{Discussion}

Radiotherapy is a key constituent of ovarian cancer treatment, which has been used for decades, with the primary limitation being the radioresistance of tumor cells. Brown (15) previously reported that hypoxic conditions lead to resistance to radiotherapy, overcoming this radioresistance would improve the efficacy of cancer radiation therapy. In addition, several other studies have focused on radioresistance of ovarian 

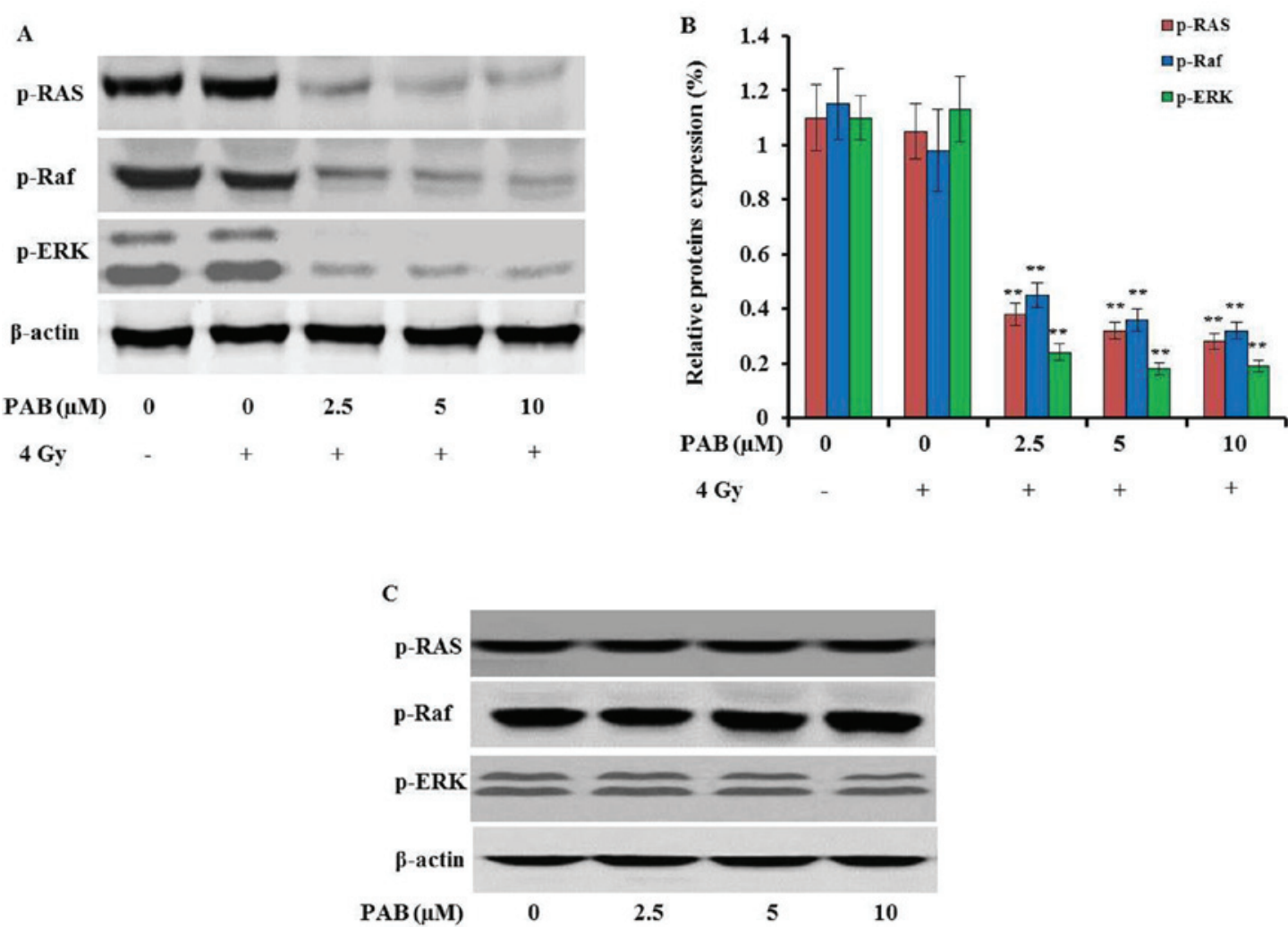

Figure 4. Combination treatment with PAB and irradiation inhibits the activation of the Ras-Raf-ERK signaling pathway. (A) SKOV-3 cells were exposed to $\mathrm{PAB}$ at varying concentrations and irradiation, and the expression of p-Ras, p-Raf, and p-ERK was analyzed by western blot analysis. (B) A densitometry analysis of the bands was performed. (C) SKOV-3 cells were exposed to PAB alone and analyzed by western blot analysis. ${ }^{* *} \mathrm{P}<0.01$ vs. 4 Gy radiation group. $\mathrm{PAB}$, pseudolaric acid B; p, phosphorylated; Raf, RAF proto-oncogene serine/threonine-protein kinase; ERK, extracellular signal-regulated kinase.

cancer $(16,17)$. The present study investigated the potential of PAB as a radiosensitizer during the irradiation of SKOV-3 ovarian cancer cells.

PAB has been demonstrated to exhibit antitumor effects on a wide variety of tumor cells, including ovarian cancer cells $(9,13)$. However, there have been few studies focused on the effects of PAB when administered alongside radiotherapy. The present study aimed to determine the potential of PAB as a radiosensitizer during the treatment of ovarian cancer, as well as investigating the underlying mechanisms by which it is able to have a radiosensitizing effect. The effectiveness of radiotherapy as a single therapy and as a combined therapy with $\mathrm{PAB}$ was studied by comparing the cell survival rates of the SKOV-3 ovarian cancer cell line when treated with each therapy type. In addition, autophagy, apoptosis and the Ras-Raf-ERK signaling pathway were studied in order to help determine the mechanism by which PAB exerts a radiosensitizing effect. Yu et al (13) reported that PAB could induce HO-8910 and A2780 ovarian cancer cell apoptosis. However, in the present study PAB had no apoptotic effect on the SKOV-3 cells. When a combined treatment of radiotherapy and $\mathrm{PAB}$ was administered, the surviving fraction of cells decreased compared to the group treated with radiotherapy alone, indicating that $\mathrm{PAB}$ is a radiosensitizing agent.

To determine the mechanisms of cell death induced by the combination of PAB and irradiation, an autophagy assay was completed using MDC, a fluorescent dye used to label autophagic vacuoles. The results demonstrated no notable change in the number of autophagic vacuoles. The expression of LC3-I, LC3-II and ATG5 was consistent with the MDC staining assay results, with no notable changes seen. These results demonstrated that autophagy was not affected by PAB. PAB treatment alone had no effect on SKOV-3 cell growth, whereas irradiation treatment alone did not induce cell autophagy either. Thus, it was hypothesized that the combination treatment would induce apoptosis in SKOV-3 cells.

Previous studies have revealed that apoptosis is a significant method of cell death during drug and irradiation treatment $(18,19)$. The increased induction of apoptosis has been investigated using several different radiosensitizers, including docetaxel (20), oxaliplatin (21) and vinorelbine (22). These previous results led to the proposal that the radiosensitizing effect of PAB may be due to it increasing the rate of apoptosis of SKOV-3 cells. The results of the Annexin V/PI staining assay supported this theory, demonstrating a significant increase in the rate of apoptosis during treatment with PAB and irradiation in a dose-dependent manner.

Raf proteins can be activated by the human proto-oncogene Ras and regulated by the expression of the downstream protein ERK (23). Ovarian cancer is the result of multiple genetic and epigenetic changes in patients. It has been reported that Ras is mutated in $>20 \%$ of cases of ovarian cancer (24) and that the Ras-Raf-ERK signaling pathway is involved in the progression of ovarian cancer, thus it represents a potential target for anticancer therapy (25). In the present study, combination therapy with PAB and irradiation notably decreased the levels of phosphorylated proteins in the Ras-Raf-ERK singling pathway when compared with the irradiation treatment alone. 
Therefore, it was hypothesized that decreasing the phosphorylation of proteins in the Ras-Raf-ERK signaling pathway could also inhibit the progression of ovarian cancer, which may due to enhanced apoptosis. In other studies, it has been reported that irradiation enhanced the interactions between Ras and Raf proteins, and the activation of ERK (26-28). In the present study, treatment with irradiation and PAB was demonstrated to lower the expression of ERK, which could be due to the combination with $\mathrm{PAB}$, but this hypothesis requires further study to be confirmed.

Meanwhile, Kyula et al (29) reported that the Ras-Raf-ERK signaling pathway not only serves a pivotal role in regulating cancer progression, but is also involved in treatment resistance. Currently, the main obstacle for radiotherapy is the degree of radioresistance. In the present study, PAB treatment alone had no effect on SKOV-3 cells, whereas combination therapy could inhibit the Ras-Raf-ERK signaling pathway. This suggests that combination therapy with PAB and irradiation not only inhibited the progression of the ovarian cancer, but also reduced irradiation therapy resistance by regulating the activity of the Ras-Raf-ERK signaling pathway.

In conclusion, the combination of PAB with irradiation increased the efficiency of radiation therapy in SKOV-3 cells by inhibiting the Ras-Raf-ERK signaling pathway and increasing cell apoptosis. This indicates that the downregulation of the Ras-Raf-ERK signaling pathway is not only involved in inhibiting tumor progression, but also in reducing irradiation resistance. However, this hypothesis requires further research to be confirmed. The results of the present study indicate that $\mathrm{PAB}$ has therapeutic potential as a novel radiosensitizer for the treatment of ovarian cancer.

\section{Acknowledgements}

The present study was supported by the Natural Science Foundation of Shandong Province (grant no. ZR2014YL029).

\section{References}

1. Jemal A, Siegel R, Ward E, Hao Y, Xu J, Murray T and Thun MJ: Cancer statistics, 2008. CA Cancer J Clin 58: 71-96, 2008.

2. Goff BA, Mandel L, Muntz HG and Melancon CH: Ovarian carcinoma diagnosis. Cancer 89: 2068-2075, 2000.

3. Bast RC Jr, Hennessy B and Mills GB: The biology of ovarian cancer: New opportunities for translation. Nat Rev Cancer 9: 415-428, 2009.

4. Bookman MA, Brady MF, McGuire WP, Harper PG, Alberts DS, Friedlander M, Colombo N, Fowler JM, Argenta PA, De Geest K, et al: Evaluation of new platinum-based treatment regimens in advanced-stage ovarian cancer: A Phase III Trial of the Gynecologic Cancer Intergroup. J Clin Oncol 27: 1419-1425, 2009.

5. Schwarz R, Bruland O, Cassoni A, Schomberg P and Bielack S: The role of radiotherapy in oseosarcoma. Cancer Treat Res 152 147-164, 2009.

6. Zhang YC, Jiang G, Gao H, Liu HM and Liang J: Influence of ionizing radiation on ovarian carcinoma SKOV-3 xenografts in nude mice under hypoxic conditions. Asian Pac J Cancer Prev 15: 2353-2358, 2014.

7. Di Pasqua AJ, Yuan H, Chung Y, Kim JK, Huckle JE, Li C, Sadgrove M, Tran TH, Jay M and Lu X: Neutron-activatable holmium-containing mesoporous silica nanoparticles as a potential radionuclide therapeutic agent for ovarian cancer. J Nucl Med 54: 111-116, 2013.

8. Wakeford R: The cancer epidemiology of radiation. Oncogene 23: 6404-6428, 2004.
9. Yu JH, Cui Q, Jiang YY, Yang W, Tashiro S, Onodera S, Onodera S and Ikejima T: Pseudolaric acid B induces apoptosis, senescence, and mitotic arrest in human breast cancer MCF-7. Acta Pharmacol Sin 28: 1975-1983, 2007.

10. Li E, Clark AM and Hufford CD: Antifungal evaluation of pseudolaric acid B, a major constituent of Pseudolarix kaempferi. J Nat Prod 58: 57-67, 1995.

11. Wang WC, Lu RF, Zhao SX and Gu ZP: Comparison of early pregnancy-terminating effect and toxicity between pseudolaric acids A and B. Zhongguo Yao Li Xue Bao 9: 445-448, 1988 (In Chinese).

12. Wang WC, Lu RF, Zhao SX and Zhu YZ: Antifertility effect of pseudolaric acid B. Zhongguo Yao Li Xue Bao 3: 188-192, 1982 (In Chinese).

13. Yu B, Yue DM, Shu LH, Li NJ and Wang JH: Pseudolaric acid B induces caspase-dependent cell death in human ovarian cancer cells. Oncol Rep 31: 849-857, 2014.

14. Morimoto H, Yonehara S and Bonavida B: Overcoming tumor necrosis factor and drug resistance of human tumor cell lines by combination treatment with anti-Fas antibody and drugs or toxins. Cancer Res 53: 2591-2596, 1993.

15. Brown JM: Tumor hypoxia in cancer therapy. Methods Enzymol 435: 297-321, 2007.

16. Steffen AC, Gostring L, Tolmachev V, Palm S, Stenerlow B, Carlsson J: Differences in radiosensitivity between three HER2 overexpressing cell lines. Eur J Nucl Med Mol Imaging 35: 1179-1191, 2008.

17. Akbarzadeh M, Nouri M, Banekohal MV, Cheraghi O, Tajalli H, Movassaghpour A, Soltani S, Cheraghi H, Feizy N, Montazersa heb $\mathrm{S}$, et al: Effects of combination of melatonin and laser irradiation on ovarian cancer cells and endothelial lineage viability. Lasers Med Sci 31: 1565-1572, 2016.

18. Pauwels B, Vermorken JB, Wouters A, Ides J, Van Laere S, Lambrechts HA J, Pattyn GG, Vermeulen K, Meijnders P and Lardon F: The role of apoptotic cell death in the radiosensitising effect of gemcitabine. Br J Cancer 101: 628-636, 2009.

19. Mose S, Class R, Weber HW, Rahn A, Brady LW and Bottcher HD: Radiation enhancement by gemcitabine-mediated cell cycle modulations. Am J Clin Oncol 26: 60-69, 2003.

20. Creane M, Seymour CB, Colucci S and Mothersill C: Radiobiological effects of docetaxel (Taxotere): A potential radiation sensitizer. Int J Radiat Biol 75: 731-737, 1999.

21. Hermann RM, Rave-Frank M and Pradier O: Combining radiation with oxaliplatin: A review of experimental results. Cancer Radiother 12: 61-67, 2008.

22. Zhang M, Boyer M, Rivory L, Hong A, Clarke S, Stevens G and Fife K: Radiosensitization of vinorelbine and gemcitabine in NCI-H460 non-small-cell lung cancer cells. Int J Radiat Oncol Biol Phys 58: 353-360, 2004.

23. Maurer G, Tarkowski B and Baccarini M: Raf kinases in cancer-roles and therapeutic opportunities. Oncogene 30: 3477-3488, 2011.

24. Kurman RJ and Shih IeM: Pathogenesis of ovarian cancer: Lessons from morphology and molecular biology and their clinical implications. Int J Gynecol Pathol 27: 151-160, 2008.

25. Yap TA, Carden CP and Kaye SB: Beyond chemotherapy: Targeted therapies in ovarian cancer. Nat Rev Cancer 9: 167-181, 2009.

26. Kasid U and Dritschilo A: RAF antisense oligonucleotide as a tumor radiosensitizer. Oncogene 22: 5876-5884, 2003.

27. Suy S, Anderson WB, Dent P, Chang E and Kasid U: Association of Grb2 with Sos and Ras with Raf-1 upon gamma irradiation of breast cancer cells. Oncogene 15: 53-61, 1997.

28. Sylvain V, Lafarge S and Bignon YJ: Molecular pathways involved in response to ionizing radiation of ID-8 mouse ovarian cancer cells expressing exogenous full-length Brcal or truncated Brcal mutant. Int J Oncol 19: 599-607, 2001.

29. Kyula JN, Khan AA, Mansfield D, Karapanagiotou EM, McLaughlin M, Roulstone V, Zaidi S, Pencavel T, Touchefeu Y, Seth R, et al: Synergistic cytotoxicity of radiation and oncolytic Lister strain vaccinia in (V600D/E)BRAF mutant melanoma depends on JNK and TNF- $\alpha$ signaling. Oncogene 33: 1700-1712, 2014.

This work is licensed under a Creative Commons Attribution-NonCommercial-NoDerivatives 4.0 International (CC BY-NC-ND 4.0) License. 